\title{
Efficacy of cystectasia in the treatment of ketamine-induced bladder contracture
}

\author{
Xue-Song Yang ${ }^{1,2 \#}$, Zheng Chen ${ }^{1 \#}$, Jian-Li Duan ${ }^{3}$, Bin Pan ${ }^{1}$, Xiao-Ping Qin ${ }^{1}$, Bin Lei ${ }^{1}$, Yang-Bai Lu ${ }^{1}$, \\ Yu-Tong Li ${ }^{1}$, Yun Luo ${ }^{3}$, Xiao-Long Xu ${ }^{4}$, Cai-Yong Lai ${ }^{1}$, Yu-Min Zhuo ${ }^{1}$ \\ ${ }^{1}$ Department of Urology, The First Affiliated Hospital of Jinan University, Guangzhou 510632, China; ${ }^{2}$ Department of Urology, Affiliated Hospital \\ of North Sichuan Medical College, Nanchong 637000, China; ${ }^{3}$ Department of Urology, The Third Affiliated Hospital of Sun Yat-sen University, \\ Guangzhou 510630, China; ${ }^{4}$ Department of Urology, The Third People's Hospital of Dongguan, Guangdong, Dongguan 523320, China \\ Contributions: (I) Conception and design: YM Zhuo, Z Chen; (II) Administrative support: XS Yang, Z Chen; (III) Provision of study materials or \\ patients: B Pan, XP Qin, B Lei; (IV) Collection and assembly of data: YB Lu, Y Luo, XL Xu; (V) Data analysis and interpretation: YT Li, JL Duan, \\ CY Lai; (VI) Manuscript writing: All authors; (VII) Final approval of manuscript: All authors. \\ \#These authors contributed equally to this work. \\ Correspondence to: Dr. Yu-Min Zhuo. Department of Urology, The First Affiliated Hospital of Jinan University, No. 613 Huangpu Avenue West, \\ Guangzhou 510632, China. Email: Tzhuoyumin@126.com.
}

Background: The treatment of ketamine-induced bladder contractures remains poorly studied. We therefore evaluated the efficacy of cystectasia with a sodium hyaluronate balanced solution in this kind of bladder contracture.

Methods: Eighteen patients presenting with ketamine-induced bladder contracture between July 2010 and February 2018 were selected and analysed. Ketamine was discontinued in all patients, who were then treated with weekly cystectasia $(0.09 \%$ sodium hyaluronate balanced solution) 3 times. The volume of the first perfusion was twice the preoperatively measured bladder capacity, and the volume of the subsequent two perfusions was increased by $100 \mathrm{~mL}$ each time. The Pelvic Pain and Urgency/Frequency (PUF) symptom score, O’Leary-Sant Interstitial Cystitis (IC) Symptom Index (ICSI), IC Problem Index (ICPI), Quality of Life (QOL) score, and bladder capacity were recorded before surgery and 3 and 12 months after the $3^{\text {rd }}$ expansion.

Results: No significant complications were observed during the 3 expansions. Fourteen patients completed the full follow-up schedule. Preoperatively and at the 3- and 12-month follow-up evaluations performed after the $3^{\text {rd }}$ expansion, the PUF symptom scores were 20.4 $\pm 3.6,11.5 \pm 3.1$, and $13.2 \pm 3.3$, respectively; the mean ICSI was $13.6 \pm 2.8,7.7 \pm 2.3$, and $8.2 \pm 2.5$, respectively; the mean ICPI was $10.6 \pm 2.6,7.3 \pm 2.1$, and $7.7 \pm 2.5$, respectively; and the mean QOL scores were $6.0 \pm 0,2.1 \pm 0.5$, and $2.7 \pm 0.8$, respectively; and the mean bladder catheter volume was $83 \pm 27,234 \pm 56$, and $228 \pm 52 \mathrm{~mL}$, respectively. There were significant differences between all preoperative and postoperative values.

Conclusions: Cystectasia with a sodium hyaluronate balanced solution is an effective treatment modality for ketamine-induced bladder contracture.

Keywords: Ketamine; urocystic contracture; cystectasia; sodium hyaluronate

Submitted Nov 20, 2019. Accepted for publication Mar 16, 2020.

doi: $10.21037 /$ tau.2020.04.01

View this article at: http://dx.doi.org/10.21037/tau.2020.04.01 


\section{Introduction}

Young adults and adolescents are increasingly abusing ketamine as a recreational drug (1). One of the adverse health effects of abusing this drug is ketamine cystitis, which is characterized by lower urinary tract symptoms (LUTS), such as decreased bladder volume, decreased bladder compliance, dysuria, gross haematuria, and urge incontinence. These findings and symptoms are due to the toxic effects of dihydroxybenzene, a metabolite of ketamine (2-5). In severe cases, bladder contracture and vesicoureteral reflux can occur, leading to ketamineassociated urinary dysfunction (KAUD) and even cancer (6). These pathological effects have a substantial physical and mental impact on addicts and place a huge burden on their families, communities, and nations $(7,8)$. At present, there are several treatment options for ketamine cystitis; these include the use of $\alpha$ receptors and $M$ receptor blockers for the treatment of overactive bladder, antibiotics in patients with high white blood cell counts, and augmentation cystoplasty utilizing the sigmoid colon (9). However, researchers have also discovered that some of these agents have side effects, such as constipation and blurred vision, that can lead to an unsatisfactory quality of life (QOL) (10). These shortcomings have prompted further efforts in this field. In this study, we included 18 patients who underwent treatment for a bladder capacity $<120 \mathrm{~mL}$ due to ketamine abuse in 3 hospitals from July 2010 to February 2018. We performed cystectasia by infusing patients with a balanced solution of $0.09 \%$ sodium hyaluronate and then evaluated the efficacy of this treatment.

\section{Methods}

\section{Patients}

Eighteen male patients who underwent treatment for bladder contracture induced by ketamine abuse at the Affiliated Hospital of North Sichuan Medical College, the First Affiliated Hospital of Jinan University, the Third Affiliated Hospital of Sun Yat-sen University and the Third People's Hospital of Dongguan from July 2010 to February 2018 were included. This study was approved by the ethics committees of these institutions, and all patients signed informed consent forms. The bladder capacity of the patients $<120 \mathrm{~mL}$, as measured by B-scan. The bladder capacity was defined as the vertical diameter $\times$ the longitudinal diameter $\times$ the transverse diameter $\times 0.52$. The patients ranged in age from $17-33$ years, with an average age of 24.5 years. The patients had been using ketamine for 2.14 years at a dose of 0.1-3.0 g 2-19 times per week. Based on the recorded measurements, the average preoperative bladder capacity was $74 \mathrm{~mL}$ (range, 59-119 mL), white blood cell counts ranged from ++ to ++++ on routine urine tests, and 24-hour urine protein levels ranged from 0.1-1.3 g (Table 1). Moreover, there were 5 patients $(27.78 \%)$ with an increased creatinine level and 4 patients $(22.22 \%)$ with a decreased glomerular filtration rate (GFR; $<60 \mathrm{~mL} / \mathrm{min} / 1.73 \mathrm{~m}^{2}$ ) and an elevated creatinine level. Both computerized tomography (CT) and B-scans (Figure 1) showed significantly thickened bladder walls, morphological changes, reduced bladder capacity, and varying degrees of expansion in the full or upper unilateral/bilateral collecting systems and ureters.

\section{Treatment methods}

Combined spinal-epidural anaesthesia (CSEA) was administered in the lithotomy position. An Olympus cystoscope (Japan, CYF-5A) was inserted directly into the bladder and revealed a significantly reduced bladder capacity. Next, the inferior aspect, left wall, right wall, and superior aspect of the bladder as well as the bilateral ureteral openings and trigone were examined. The water bag was placed $100 \mathrm{~cm}$ above the operating table, and the preoperative bladder capacity was measured and recorded without stress. Cystistat (a balanced solution of 0.09\% sodium hyaluronate) was administered at a pressure of $15 \mathrm{kPa}$ via a syringe into the bladder after residual urine was removed. The volume of the first perfusion was twice the preoperatively measured bladder capacity. B-scan was used to simultaneously monitor bladder capacity and bladder wall thickness. A cystoscope revealed that the bladder wall was significantly diastolic, the distance between the haemorrhagic erythema had increased, and the small vessels presented a discontinuous course, with some vessels partly disconnected or exhibiting mucosal cracking. The solution was maintained for $30 \mathrm{~min}$ and then discharged. No active bleeding was noted during cystoscopy. An F22 3 -way silicone catheter was retained, and the bladder was continuously rinsed for $48 \mathrm{~h}$. Three days after surgery, the catheter was clamped and only opened every $2 \mathrm{~h}$ to record urine output. For the latter two rounds of perfusion, the solution was directly administered via the catheter after successful CSEA. The volume of the latter two perfusions was increased by $100 \mathrm{~mL}$ each time (Figure 1). A B-scan was used for simultaneous monitoring. The $10-\mathrm{mL}$ airbag 
Table 1 Preoperative and follow-up data of 18 patients

\begin{tabular}{|c|c|c|c|c|c|c|c|c|c|c|c|}
\hline Case & $\begin{array}{l}\text { Age } \\
(y)\end{array}$ & $\begin{array}{c}\text { Addiction } \\
\text { time }(y)\end{array}$ & $\begin{array}{l}\text { Pre-bladder } \\
\text { volume (mL) }\end{array}$ & $\begin{array}{l}\text { Pre-urination } \\
\text { frequency }(d)\end{array}$ & $\begin{array}{l}\text { Urinary } \\
\text { leukocyte }\end{array}$ & $\begin{array}{c}\text { Urinary } \\
\text { erythrocyte }\end{array}$ & $\begin{array}{l}\text { Urinary } \\
\text { albumin } \\
\text { (g) }\end{array}$ & \multicolumn{2}{|c|}{3 months after surgery } & \multicolumn{2}{|c|}{12 months after surgery } \\
\hline 2 & 21 & 2.1 & 59 & 22 & ++ & +++ & 1.2 & 12 & 197 & 9 & 218 \\
\hline 5 & 20 & 3.3 & 78 & 16 & +++ & +++ & 0.5 & 10 & 257 & 9 & 285 \\
\hline 6 & 26 & 3.2 & 80 & 17 & ++ & +++ & 0.9 & Loss & Loss & Loss & Loss \\
\hline 7 & 21 & 3.6 & 103 & 18 & +++ & ++ & 0.3 & 9 & 307 & 8 & 278 \\
\hline 11 & 22 & 2.1 & 97 & 16 & +++ & +++ & 0.2 & 10 & 252 & Loss & Loss \\
\hline 12 & 26 & 2.7 & 83 & 16 & +++ & ++ & 0.5 & 9 & 341 & 9 & 312 \\
\hline 13 & 29 & 4 & 71 & 19 & ++ & +++ & 1.3 & 11 & 234 & 9 & 267 \\
\hline 14 & 33 & 2.8 & 119 & 15 & +++ & +++ & 1 & 8 & 342 & 8 & 333 \\
\hline 15 & 25 & 2.7 & 79 & 22 & ++ & +++ & 1.1 & 9 & 279 & 8 & 300 \\
\hline 16 & 25 & 2.9 & 82 & 20 & +++ & +++ & 1.2 & 10 & 300 & loss & loss \\
\hline 17 & 27 & 3.1 & 60 & 21 & +++ & +++ & 0.7 & 8 & 260 & 7 & 256 \\
\hline
\end{tabular}

++, 20-29 leukocyte or erythrocyte per high power field under the microscope; +++, 30-39 leukocyte or erythrocyte per high power field under the microscope.
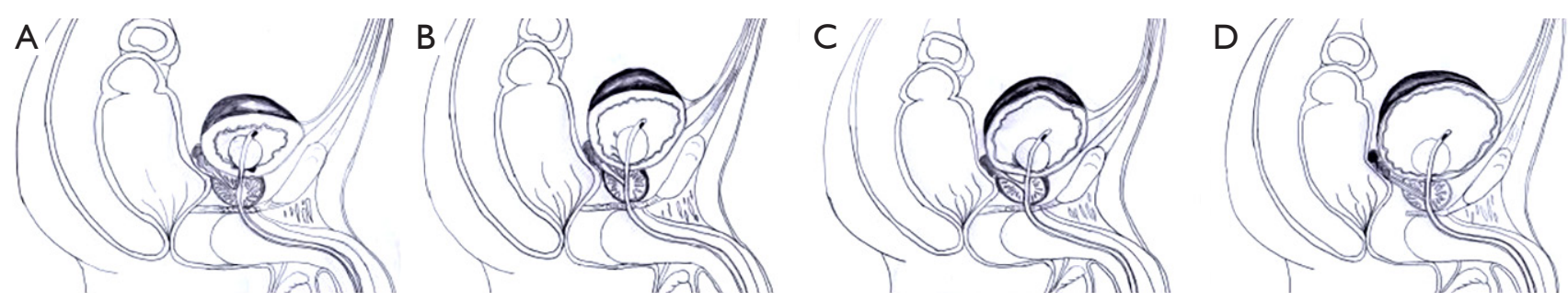

Figure 1 Contrast diagram of pre-operative and post-operative bladder. (A) Pre-operative bladder with a reduced volume and thicker wall. (B) Bladder after the first perfusion of cystistat, with its volume expanded and wall thinning. (C) Bladder after the second perfusion of cystistat, with a further expanded volume and thinner wall. (D) Bladder after the third perfusion of cystistat, showing up a much well improved bladder capacity and wall thickness. 
capacity of the catheter was included in the total volume of the solution.

\section{Follow-up}

Follow-up was carried out by questionnaire via telephone or WeChat survey to primarily record the Pelvic Pain and Urgency/Frequency (PUF) symptom score, O'Leary-Sant IC Symptom Index (ICSI), IC Problem Index (ICPI), QOL score, and bladder capacity before surgery and 3 and 12 months after the third expansion. Postoperative B-scan and CT were performed at the nearest medical facility, and the results were sent as images via WeChat.

\section{Statistical methods}

SPSS 20.0 statistical software was used to analyse the data. Continuous data with a normal distribution are shown as the mean \pm standard deviation (SD). Analysis of variance was performed using a single set of repeated measurements for comparisons between different time points within a group. Changes in bladder capacity as well as PUF, ICSI, ICPI, and QOL were assessed between before and after surgery. Categorical data are expressed as a number (percentage). $\mathrm{P}<0.05$ was regarded as statistically significant.

\section{Results}

\section{Imaging changes in the kidneys and bladders between before and after surgery}

All patients in the study showed significant imaging changes. Preoperative B-scans revealed 7, 9, and 3 cases of mild, moderate, and severe hydronephrosis, respectively, in the bilateral renal pelvis. There were 4 cases of full dilatation and 8 of upper dilatation of the bilateral ureters and 3 cases of unilateral dilatation. All 18 patients had significantly thickened bladder walls, morphologic changes, and bladder contractures and significantly reduced bladder capacities (average, $74 \mathrm{~mL}$; range, 59-119 mL; Figure 2A).

The CT scans showed varying degrees of changes in the unilateral and bilateral collecting systems and full and upper dilatation of the ureters but no evidence of calcifications or abnormal densities in the bilateral renal parenchyma. The most important showed significantly thickened bladder walls and reduced bladder capacity (average $69 \mathrm{~mL}$; range, 52-119 mL; Figure 2B). Two patients had renal papillary necrosis, enlarged abdominal aorta lymph nodes, significantly thickened bladder walls, and morphological changes. Three patients had small gourd-shaped bladders, one had a pebbleshaped bladder, and two had bladder diverticula. Moreover, there were six patients with seminal vesicle enlargement, suggesting inflammatory changes. Dynamic enhancement showed seven patients had thickened ureteral walls.

Compared to preoperative B-and CT scans, postoperative scans performed in all 14 patients showed significantly increased bladder capacities, improved bladder filling, and thinner bladder walls. B-scans obtained 3 months after surgery showed that the maximum bladder capacity was approximately $285 \mathrm{~mL}$ (average, 234 $56 \mathrm{~mL}$; Figure 2C), while CT scans obtained 3 months after surgery showed the maximum bladder capacity to be approximately $307 \mathrm{~mL}$ (average, 274 $39 \mathrm{~mL}$; Figure 2D).

\section{Urine volume and frequency, PUF, ICSI, ICPI, QOL, and $R U$ changes after surgery}

Two patients were lost to follow-up after surgery. Two patients relapsed after 8 months. Fourteen patients were followed up for at least 12 months. As shown in Figure 3, the results obtained for PUF, ICSI, ICPI, QOL score, and bladder capacity indicated that compared to preoperative values, postoperatively, the patients showed significant improvement in bladder capacity at 3 and 12 months after surgery. Before surgery and at 3 and 12 months after surgery, the PUF was $20.4 \pm 3.6,11.5 \pm 3.1$, and $13.2 \pm 3.3$, respectively; the ICSI was $13.6 \pm 2.8,7.7 \pm 2.3$, and $8.2 \pm 2.5$, respectively; the ICPI was $10.6 \pm 2.6,7.3 \pm 2.1$, and $7.7 \pm 2.5$, respectively; the QOL was $6.0 \pm 0,2.1 \pm 0.5$, and $2.7 \pm 0.8$, respectively; and bladder capacity was $83 \pm 27,234 \pm 56$, and $228 \pm 52 \mathrm{~mL}$, respectively. Statistical analysis showed that after 3 months, the PUF, ICSI, ICPI, and QOL scores were markedly better than those obtained preoperatively $(\mathrm{P}<0.001)$. There was no significant difference in the data obtained after 3 and 12 months of follow-up $(\mathrm{P}>0.05$; Figure 3).

\section{Discussion}

Ketamine is a drug that can be administered via a variety of routes and is abused among the younger generation (11). This abuse leads to bladder contractures and complex pathophysiological effects encompassing both histological inflammation and abnormal nerve regulation as well as complications associated changes in bladder morphology (12). To date, the following three theories have been advanced to 

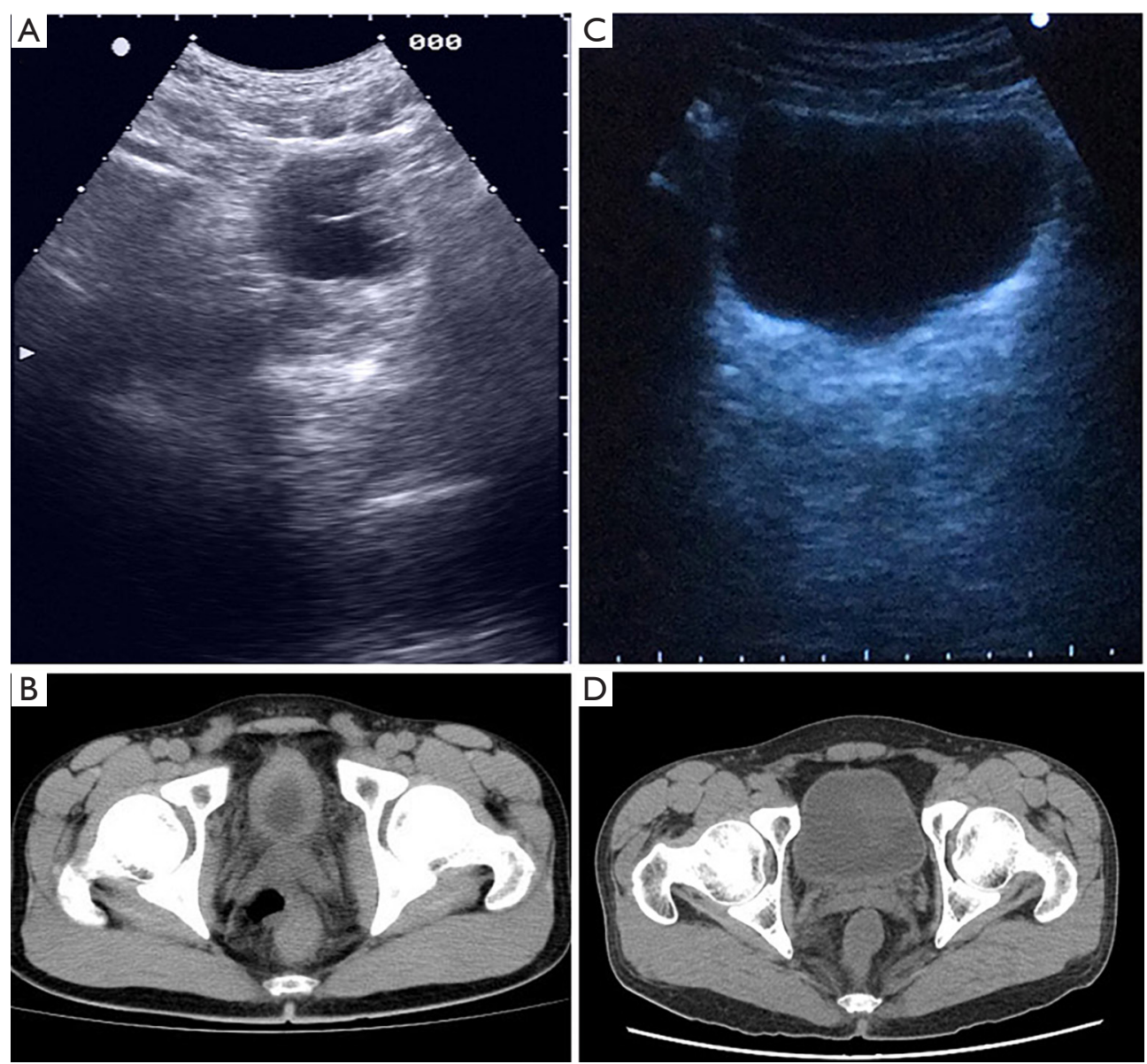

Figure 2 Comparison of a B-scan and CT scan before and after survey. (A,C) Were the preoperative and postoperative B-scan results of the same patient. (B,D) Were the preoperative and postoperative CT scan results of the same patient. (A) Preoperative B-scan of the bladder capacity measured at about $55 \mathrm{~mL}$. (B) Preoperative CT scan of the bladder capacity measured at about $52 \mathrm{~mL}$. (C) B-scan taken 3 months after surgery, of the bladder capacity measured at about $285 \mathrm{~mL}$. (D) CT scan taken 3 months after surgery, of the bladder capacity measured at about $307 \mathrm{~mL}$. Bladder capacity was defined as: vertical diameter $\times$ longitudinal diameter $\times$ transverse diameter $\times 0.52$.

explain the mechanisms underlying the damage associated with ketamine $(7,13)$. (I) Ketamine or its metabolites damage the protective layer on the bladder mucosal surface and thereby impairs the epithelial permeability barrier as well as the deep tissues in the bladder wall. Subsequently, infiltration by ketamine or its metabolites into the submucosal and detrusor layers can stimulate nerve fibres on the bladder wall, resulting in decreased bladder volume and compliance. (II) High concentrations of ketamine or its metabolites induce nonspecific interstitial inflammation related to autoimmune responses to the bladder urinary tract epithelium and submucosa, resulting in the activation of the complement system and the upregulation of C3/ $\mathrm{C} 4$ and an increase in the erythrocyte sedimentation rate. These changes can cause immersion, activation, and degranulation of the mast cells and increase the release of histamine, leading to vasodilation, congestion, inflammatory cell exudation, chemotaxis, and the stimulation of Group $\mathrm{C}$ nerve fibres. These effects are followed by the release of neuropeptides and other chemical neurotransmitters and consequential effects on inflammatory responses and damage to bladder tissues. (III) Ketamine or its metabolites damage endothelial cells in the bladder and kidney capillaries, leading to decreased endothelial capillary density, hardened blood vessel walls, and microcirculatory disorders. When these conditions are aggravated, the resulting bladder mucosal ischaemia can cause interstitial fibrosis and decrease bladder capacity, while renal papilla 


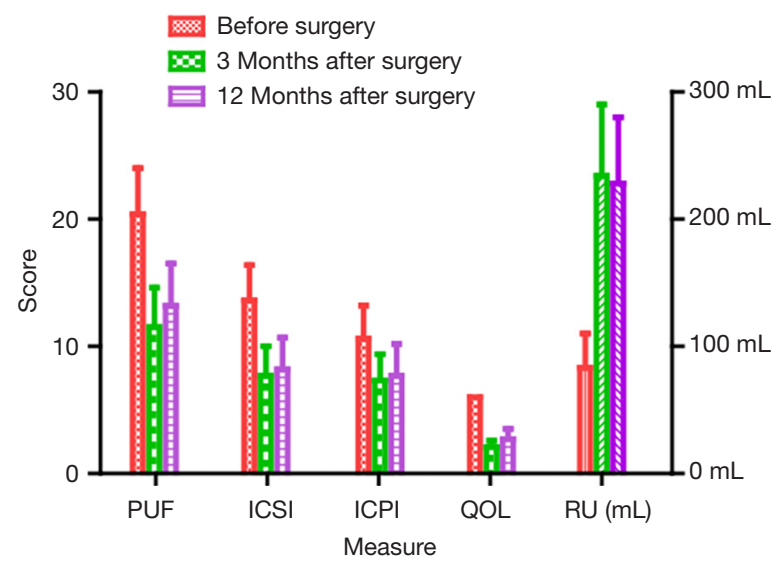

Figure 3 Comparison of PUF, ICSI, ICPI, QOL and RU before and after surgery follow-up after 3 rounds of treatments in these 14 patients. PUF, The Pelvic Pain and Urgency/Frequency symptom score; ICSI, O’Leary-Sant IC Symptom Index; ICPI, IC Problem Index, QOL, quality of life.

sclerosis or microvascular diseases in the renal medulla can cause a lack of blood perfusion in the renal papillae, which may eventually progress into renal tubular-interstitial nephropathy and renal cortical atrophy, although this latter effect needs further verification. Therefore, long-term use of high doses of ketamine can induce effects that damage the upper urinary tract and lead to thickening of the bladder wall due to the direct toxicity of ketamine $(14,15)$.

Imaging changes in the urinary system caused by ketamine abuse manifest as LUTS, which are clearly visible on B-scans, CT scans, and intravenous pyelograms (IVPs). The B-scan and CT findings presented in this study are consistent with previously reported changes in the urinary system (16) (Figure 1A,C) and included significant thickening and contracture of the bladder wall. Although no calcifications were observed, bladder capacity was significantly reduced, with the most severe case having a capacity of only $48 \mathrm{~mL}$. For some patients, imaging performed at 3 months after cystectasia (Figure 1B,D) revealed that the urinary systems were significantly better than their preoperative state and exhibited good bladder filling, smooth bladder walls, and a capacity of $>250 \mathrm{~mL}$. During data collection, we also found that creatinine levels were elevated in 4 patients $(22.22 \%)$ due to ureteral reflux and subsequent obstructive injury due to the longterm reduction in bladder capacity; however, the authors speculate that the actual probability of renal damage was higher in this group because the GFR is a more sensitive indicator than serum creatinine in evaluating renal function. The GFR was directly proportional to the duration of use and the amount of ketamine taken in approximately $50 \%$ of the patients in the current study. Nevertheless, we maintain that the normal creatinine levels and GFRs recorded in the other $50 \%$ of patients did not necessarily indicate intact renal function. Because GFR detection can be used to identify cases in which the kidneys are damaged due to ketamine abuse, the GFR is recommended as a routine clinical test for patients using ketamine.

During the diagnosis and treatment process, we found that bladder contractures often presented clinically with storage symptoms and that acute onset could be complicated by persistent haematuria and severe suprapubic pain. This was the main reason that patients sought evaluation at the hospital. We found that most patients had mental disorders and sexual dysfunction, which was impossible to restore due to the LUTS caused by bladder contracture (17). Since early approaches performed using hydrodistension, the clinical solution to this problem had progressed through practice $(18,19)$. Cheng et al. (20) reported that making a radial reticular incision in the bladder using a green light laser achieved favourable results with regard for expansion in China. The excellent haemostatic effect achieved by the laser was effective in minimizing the incidence of severe bleeding; however, further hydrodistension was still necessary in patients with a bladder capacity $<50 \mathrm{~mL}$. To prevent the bladder from rupturing due to water expansion, it should be noted that the water pressure should not exceed $20 \mathrm{kPa}$ during the operation, which should be performed under anaesthesia. If a tear in the bladder detrusor layer is seen on cystoscopy, the expansion can be stopped. During the expansion process, the sodium hyaluronate solution could be quickly absorbed by the bladder mucosa at the tear site, and this could effectively increase the bladder capacity and thereby improve the urinary tract symptoms associated with ketamine.

We believe that the main cause of ketamine-associated cystitis is bladder contracture. Due to the obvious fibrosis of the bladder muscle layer, the primary task of treatment is to expand the bladder volume. The use of saline expansion under anaesthesia is a highly effective method. The fibrotic muscle layer could be torn to expand the bladder, and this procedure can be repeated many times. Indeed, a onestage operation is insufficient for achieving a desirable expansion outcome (21). Our treatment process included initial bladder perfusion, bladder hydrodistension, and bladder augmentation followed by cystectasia with sodium hyaluronate solution. Treatment options and timing should typically be determined on the basis of each patient's dose, 
duration, bladder capacity, and general condition (22). Among the 18 patients enrolled in this study, the followup evaluation performed 1 month after 3 rounds of cystectasia showed that single urine output was increased by 5 -fold compared to the output at preoperative levels, and the frequency of urination decreased from once every $30 \mathrm{~min}$ to nearly once every $120 \mathrm{~min}$. Thus, bladder capacity was effectively increased and urine frequency effectively lowered, significantly improving the LUTS. We hypothesized that during the expansion process, the increased bladder pressure disrupted the detrusor's nerve endings that receive sensory input, which leads to ischaemic necrosis of the sensory nerves on the bladder wall so that the distribution and density of the sensory nerves decreased. In addition, because the sodium hyaluronate solution is rich in glucuronic acid, it could potentially prevent the production of some of the enzymes in the cells and diminish free radical formation. Low-molecular weight hyaluronic acid is thought to exert anti-inflammatory effects, inhibits pathogens, has strong penetrability and is also effective in promoting the growth and repair of the bladder mucosa; however, these hypotheses warrant further testing.

In summary, our study shows that cystectasis is a safe and effective procedure that improves the LUTS and KAUD caused by ketamine-induced bladder contractures that requires only a simple procedure and causes minimal injury.

\section{Acknowledgments}

Funding: This work is supported by the following grants: Leading Specialist Construction Project-Department of Urology, the First Affiliated Hospital, Jinan University (No. 711006). Postdoctoral Fund of the First Affiliated Hospital, Jinan University (No. 809011). Postdoctoral Program of the International Training Program for Outstanding Scientific Research of Guangdong Province [2019]. National Natural Science Foundation of China (No. 81902615), Science and Technology Planning Project of Guangzhou (No. 201610010016), Young Teacher Training Program of Sun Yat-sen University (15ykpy27).

\section{Footnote}

Conflicts of Interest: All authors have completed the ICMJE uniform disclosure form (http://dx.doi.org/10.21037/ tau.2020.04.01). The authors have no conflicts of interest to declare.
Ethical Statement: The authors are accountable for all aspects of the work and ensuring that questions related to the accuracy or integrity of any part of the work are appropriately investigated and resolved. The study was approved by the Clinical Research Approval Document from the Ethics Committee of Clinical Medicine Research of the Third Affiliated Hospital of Sun Yat-sen University $\{$ No. [2010]2-130\}. And all patients signed informed consent forms.

Open Access Statement: This is an Open Access article distributed in accordance with the Creative Commons Attribution-NonCommercial-NoDerivs 4.0 International License (CC BY-NC-ND 4.0), which permits the noncommercial replication and distribution of the article with the strict proviso that no changes or edits are made and the original work is properly cited (including links to both the formal publication through the relevant DOI and the license). See: https://creativecommons.org/licenses/by-nc-nd/4.0/.

\section{References}

1. Fan N, Xu K, Ning Y, et al. Profiling the psychotic, depressive and anxiety symptoms in chronic ketamine users. Psychiatry Res 2016;237:311-5.

2. Becerra L, Schwartzman RJ, Kiefer RT, et al. CNS Measures of Pain Responses Pre- and Post-Anesthetic Ketamine in a Patient with Complex Regional Pain Syndrome. Pain Med 2015;16:2368-85.

3. Kidger E, Stahlschmidt J, Garthwaite M, et al. A Rare Urachal Cyst in a Case of Ketamine-induced Cystitis Provides Mechanistic Insights. Urology 2016;90:223.e1-7.

4. Craven R. Ketamine. Anaesthesia 2007;62 Suppl 1:48-53.

5. Wai MS, Luan P, Jiang Y, et al. Long term ketamine and ketamine plus alcohol toxicity - what can we learn from animal models? Mini Rev Med Chem 2013;13:273-9.

6. Oxley JD, Cottrell AM, Adams S, et al. Ketamine cystitis as a mimic of carcinoma in situ. Histopathology 2009;55:705-8.

7. Shahani R, Streutker C, Dickson B, et al. Ketamineassociated ulcerative cystitis: a new clinical entity. Urology 2007;69:810-2.

8. Wu P, Wang Q, Huang Z, et al. Clinical staging of ketamine-associated urinary dysfunction: a strategy for assessment and treatment. World J Urol 2016;34:1329-36.

9. Fang LK, Zhang ZJ, Yang JG. Treatment of contracted bladder from ketamine abuse with sigmoid cystoplasty. 
Chinese Journal of Urology 2010;31:471-4.

10. Ko IG, Moon BM, Kim SE, et al. Effects of Combination Treatment of Alpha 1-Adrenergic Receptor Antagonists on Voiding Dysfunction: Study on Target Organs in Overactive Bladder Rats. Int Neurourol J 2016;20:S150-8.

11. Middela S, Pearce I. Ketamine-induced vesicopathy: a literature review. Int J Clin Pract 2011;65:27-30.

12. McGinn KA, Bishop L, Sarwal A. Use of Ketamine in Barbiturate Coma for Status Epilepticus. Clin Neuropharmacol 2016;39:62-5.

13. Wei YB, Yang JR, Yin Z, et al. Genitourinary toxicity of ketamine. Hong Kong Med J 2013;19:341-8.

14. Chu PS, Ma WK, Wong SC, et al. The destruction of the lower urinary tract by ketamine abuse: a new syndrome? BJU Int 2008;102:1616-22.

15. Lai Y, Wu S, Ni L, et al. Ketamine-associated urinary tract dysfunction: an underrecognized clinical entity. Urol Int 2012;89:93-6.

16. Mason K, Cottrell AM, Corrigan AG, et al. Ketamineassociated lower urinary tract destruction: a new radiological challenge. Clin Radiol 2010;65:795-800.

17. Ng CM, Ma WK, To KC, et al. The Chinese version of the pelvic pain and urgency/frequency symptom scale: a useful assessment tool for street-ketamine abusers

Cite this article as: Yang XS, Chen Z, Duan JL, Pan B, Qin XP, Lei B, Lu YB, Li YT, Luo Y, Xu XL, Lai CY, Zhuo YM. Efficacy of cystectasia in the treatment of ketamine-induced bladder contracture. Transl Androl Urol 2020;9(3):1244-1251. doi:10.21037/tau.2020.04.01 with lower urinary tract symptoms. Hong Kong Med J 2012;18:123-30.

18. Zeng J, Lai H, Zheng D, et al. Effective treatment of ketamine-associated cystitis with botulinum toxin type a injection combined with bladder hydrodistention. J Int Med Res 2017;45:792-7.

19. Yee CH, Lai PT, Lee WM, et al. Clinical Outcome of a Prospective Case Series of Patients With Ketamine Cystitis Who Underwent Standardized Treatment Protocol. Urology 2015;86:236-43.

20. Cheng B, Qiu X, Li H, et al. The safety and efficacy of front-firing green-light laser endoscopic en bloc photoselective vapo-enucleation of non-muscle-invasive bladder cancer. Ther Clin Risk Manag 2017;13:983-8.

21. Bao JM, Tan WL, Wang BW, et al. Transurethral frontfiring Greenlight bladder autoaugmentation for bladder contracture: technique and clinical outcomes. Int Urol Nephrol 2016;48:475-80.

22. Gregoire MC, MacLellan DL, Finley GA. A pediatric case of ketamine-associated cystitis (Letter-to-the-Editor RE: Shahani R, Streutker C, Dickson B, et al: Ketamineassociated ulcerative cystitis: a new clinical entity. Urology 69: 810-812, 2007). Urology 2008;71:1232-3. 